\title{
Preface to Selected Papers from ACIS 2016
}

\section{John Lamp}

Editor in Chief

AJIS is very pleased to present four papers selected from ACIS 2016, held at the University of Wollongong, and expanded for journal publication

Janak Adhikari, Anuradha Mathrani, Chris Scogings, "A Longitudinal Journey with BYOD Classrooms: Issues of Access, Capability and Outcome Divides"

We have conducted a longitudinal study to investigate a BYOD initiative by a New Zealand School. This study shares rich insights in the context of technology-mediated pedagogies and specifically BYOD classroom, as to how digital divides moved beyond access and skills to ensure inclusive learning outcomes. As a part of the five-year study of the technology-mediated teaching and learning initiative, we have been able to explain some of the unanswered questions around the issue of digital divides in the learning process. We investigated issues pertaining to digital divide in the context of BYOD classrooms to make the following revelations. First, the BYOD classroom initiative did not end up accentuating existing gaps in access to digital tools and technologies, despite earlier studies indicating towards increase in gaps. Second, our analysis strongly indicated the changing nature of digital divides with the presence of gaps in terms of information literacy and critical thinking ability, as the BYOD classroom progressed to mature stage. This was eventually bridged in the later stage, as students slowly adjusted to the classroom curricular structures in the BYOD classroom. Third, learner self-efficacy has been identified as a determinant of learning outcomes. In the earlier phase of ICT adoption, learner self-efficacy is influenced by a combination of information literacy, critical thinking ability, and positive motivation; however subsequently, self-efficacy influences affordances in various aspects of social cognitive abilities related to individual's learning activities affecting how learners engage and apply technology to achieve learning outcomes.

Philip Dobson, Paul Jackson, "Using critical realism and reflexivity to explain broadband nonadoption in rural Australia"

Australia is conducting a substantial nationwide provision of broadband. It is primarily a fixed line network but includes wireless and satellite networks in more remote areas. The rollout is under the control of the NBN Co, whose goal is ensuring access to fast broadband for all Australians. The NBN Co has recently recognized the importance of adoption by including premises activated as a KPI alongside service provision. Coverage and adoption reflect the two faces of the NBN rollout - as a technical program and as a social program. Adoption will be the ultimate measure of the success of the NBN as a social program. Ubiquitous Internet adoption across all sectors is necessary to maximize the promised benefits. The adoption of broadband is an important first step in achieving this. International experience suggests that broadband adoption is plateauing and this has prompted our proposal that there needs to be focus on nonadoption rather than adoption. We suggest that it is important to be able to explain the mechanisms by which individuals respond to the promise of the Internet. Only by so doing can we address issues. We contend that there needs to be more focus on those disenchanted or disinterested "non-users" who are never likely to adopt without specific targeted strategies. We argue for a critical realist perspective, more particularly reflexivity, to better represent the adoption context and to provide a grounding for explanations of the causes behind the decision not to adopt. We also propose possible common-sense strategies to reverse non-adoption.

Agata McCormac, Dragana Calic, Marcus Butavicius, Kathryn Parsons, Tara Zwaans, Malcolm Pattinson, "A Reliable Measure of Information Security Awareness and the Identification of Bias in Responses" 
The Human Aspects of Information Security Questionnaire (HAIS-Q) is designed to measure Information Security Awareness. More specifically, the tool measures an individual's knowledge, attitude, and self-reported behaviour relating to information security in the workplace. This paper reports on the reliability of the HAIS-Q, including test-retest reliability and internal consistency. The paper also assesses the reliability of three preliminary over-claiming items, designed specifically to complement the HAISQ, and identify those individuals who provide socially desirable responses. A total of 197 working Australians completed two iterations of the HAIS-Q and the over-claiming items, approximately 4 weeks apart. Results of the analysis showed that the HAIS-Q was externally reliable and internally consistent. Therefore, the HAIS-Q can be used to reliably measure information security awareness. Reliability testing on the preliminary over-claiming items was not as robust and further development is required and recommended. The implications of these findings mean that organisations can confidently use the HAIS-Q to not only measure the current state of employee information security awareness within their organisation, but they can also measure the effectiveness and impacts of training interventions, information security awareness programs and campaigns. The influence of cultural changes and the effect of security incidents can also be assessed.

Meng Xiao, Rob Meredith, Shijia Gao, "An Exploratory Study Investigating How and Why Managers Use Tablets to Support Managerial Decision-Making"

Managers are often mobile and a large proportion of their work is dealing with decisions. Although many managers currently use tablet computers in their work, there is little research on the use of tablets for managerial decision-support. This exploratory study aims to investigate the ways in which managers use tablets to support their decision-making and the reasons why they do so. Using Task-Technology Fit theory, semi-structured interviews were conducted with 20 managers, 17 of whom used tablets for their work-related decision-making. The study reveals managers' tablet usage patterns in terms of location, tablet applications, decision activities and types. This study has also found that a range of tablet characteristics and decision-task characteristics affect managers' use of tablets to support decision-making at work. This exploratory study contributes to both academia and industry by providing evidence on the tablet decision-support area, and affording organisations, tablet vendors and tablet application developers informative findings for further improvement in the provision of tablet-based decision support.

Being able to provide the authors of these papers with a wider audience is a service that this journal values as a means of promoting their research.

Copyright: (C) 2017 Lamp. This is an open-access article distributed under the terms of the Creative Commons Attribution-NonCommercial 3.0 Australia License, which permits noncommercial use, distribution, and reproduction in any medium, provided the original author and AJIS are credited. 


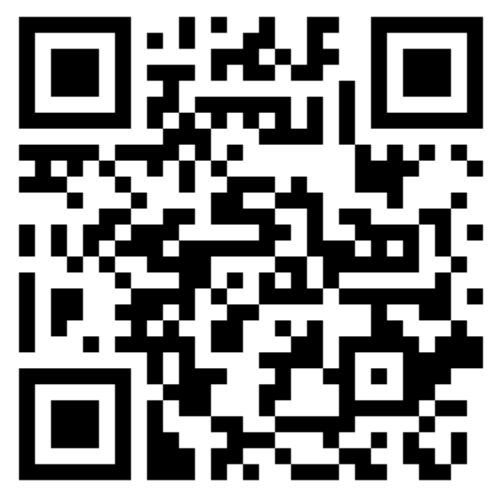

